\title{
Particle acceleration around SNR shocks
}

\author{
G. Morlino \\ INAF-Osservatorio Astrofisico di Arcetri, Largo E. Fermi, 5, 50125 Firenze, Italy
}

\begin{abstract}
We review the basic features of particle acceleration theory around collisionless shocks in supernova remnants (SNRs). We show how non linear effects induced by the back reaction of accelerated particles onto the shock dynamics are of paramount importance to 'support the hipotesys that SNRs are the factories of Galactic cosmic rays. Recent developments in the modeling of the mechanism of diffusive shock acceleration are discussed, with emphasis on the role of magnetic field amplification and the presence of neutrals in the circumstellar environment. Special attention will be devoted to observational consequences of non linear effects on the multi-wavelength spectrum of SNRs, with emphasis on X-ray and gamma-ray emission. Finally we also discuss how Balmer lines, 'detected from several young SNRs, can be used to estimate the shock dynamical properties and the efficiency of CR acceleration.
\end{abstract}

Keywords: acceleration of particles, shocks, supernova remnants, cosmic rays

\section{Introduction}

The idea that supernova remnants (SNRs) can generate the bulk of cosmic rays (CRs) observed at Earth is mainly supported by the fact that from the energetic point of view SNRs are the only class of sources in the Galaxy that can provide enough energy to explain the observed flux of CRs. Clearly the 'energetic argument alone cannot provide a proof for this $S N R$ paradigm. The basic requirements to be fulfilled are: 1) that SNRs should convert a fraction around $10-20 \%$ of their explosion energy into CRs; 2) that the spectrum individual elements and the consequent all-particle spectrum are well reproduced; 3) that the chemical abundances of nuclei are well described; 4) that the multi-frequency observation (from the radio to the gamma-ray band) of individual SNRs are well described; 5) that the anisotropy observed in the CR arrival direction is compatible with the distribution of SNRs in the Galaxy. All these points are subject to current research activities aimed to fit them in a coherent theoretical framework explaining both particle accel'eration and propagation in the Galaxy.

In this work we underline the main features of the mechanism of particle acceleration that is thought to work in SNRs ( $\$ 2$, namely the diffusive shock acceleration in its non linear version (NLDSA). Than we will analyze how some observations of SNRs could be used to infer properties on the acceleration mechanism $(\$ 3)$. In particular we will focus on three different wavebands: ( $\$ 3.1$ ) detection of thin X-ray filaments; $(\$ 3.2)$ observation of gamma-ray spectrum; $(\$ 3.3)$ Balmer lines produced by SNR shocks wich expand in partially ionized medium.

Email address: morlino@arcetri.astro.it (G. Morlino)

\section{Basic features of NLDSA}

The acceleration mechanism that is usually assumed to work in SNRs is diffusive shock acceleration (DSA)[1, 2] where particles are scattered back and forward accross the shock surface by magnetic turbulence, gaining energy at each cycle. In the so called test-particle limit, where accelerated particles are assumed to be dynamically unimportant, the theory predicts a spectrum of accelerated particles wich is a power law in energy $\propto E^{-\gamma}$ where the index $\gamma=(r+2) /(r-1)$ depends only on the compression factor, $r$, of the shock and does not depend on the details of the particle scattering process. Strong shocks in monoatomic gas has $r=4$, giving $\gamma=2$.

However the energetic requirement that at least $~ 10-20 \%$ of the kinetic energy of the supernova shell is converted to CRs leads to realize immediately that the standard test-particle version of the theory is not applicable to the description of CR acceleration. The reaction of accelerated particles onto the accelerator cannot be neglected and in fact it is responsible for spectral features that may represent potential signatures of $\mathrm{CR}$ acceleration.

There is another, possibly more important reason why DSA must include the reaction of accelerated particles: the standard diffusion coefficient typical of the interstellar medium (ISM) only leads to maximum energies of CRs in the range of $\sim \mathrm{GeV}$, rather than $\sim 10^{6} \mathrm{GeV}$ (around the knee) required by observations. A possible way to solve this inconsistency is through the plasma instabilities induced by the same accelerated particles. In fact these instabilities can generate the magnetic field structure on which particles may scatter[3], thereby reducing the acceleration time and reach larger values of the maximum energy. These plasma instabilities can be effective only if a non negligible fraction of energy is carried by high energy particles.

The main instability which is thought to be effective is the streaming instability induced by CRs which leads to magnetic 
field amplification where a spatial gradient of CRs exists. In our case a CR gradient develops upstream of the shock, hence here the magnetic field is amplified. In the absence of damping and if only resonant streaming instability is excited, the strength of the amplified magnetic field can be estimated as a function of the initial field $B_{0}$ (by using an extrapolation of quasi-linear theory) as

$$
\delta B \approx B_{0} \sqrt{2 M_{A} \xi_{c r}},
$$

where $M_{A}$ is the Alfvènic Mach number and $\xi_{c r}=P_{c r} /\left(\rho_{0} u_{s h}^{2}\right)$ is the pressure of CRs at the shock divided by the total ram pressure. For typical values of $M_{A} \sim 1000$ and $\xi_{c r} \simeq 0.20$ we get $\delta B / B_{0} \sim 20$ and the field is further amplidied by the compression when the fluid element crosses the shock from upstream to downstream.

These two processes, dynamical reaction of accelerated particles and magnetic field amplification, are the two most important ones to take into account when describing non-linear particle acceleration in SNR shocks. In the last years many authors have developed a non linear version of DSA including the back reaction of CRs (see Ref.[4] for a review) and more recently also the self-generation of magnetic field has been included self-consistently [5, 6]. In the next section we illustrate some important predictions of NLDSA, comparing them with observations of SNRs in X-ray, gamma-ray and optical $\mathrm{H} \alpha$ emission.

\section{Observational evidences of efficient $\mathbf{C R}$ acceleration}

\subsection{Non-thermal X-ray filaments}

Non-thermal X-ray radiation is produced in SNRs through synchrotron emission of high energy electrons in the magnetic field around the shock. The emission is dominated by the region downstream of the shock where the magnetic field is stronger and is cut off at a frequency that, in the case of Bohm diffusion, is independent of the strength of the magnetic field:

$$
v_{\max } \approx 0.2 u_{8}^{2} \mathrm{keV},
$$

where $u_{8}=u_{s h} /\left(10^{8} \mathrm{~cm} / \mathrm{s}\right)$ is the shock velocity in units of 1000 $\mathrm{km} / \mathrm{s}$. The maximum energy of accelerated electrons depends on the strength of the amplified magnetic field and can be estimated as

$$
E_{\max } \approx 10 B_{100}^{-1 / 2} u_{8} \mathrm{TeV},
$$

where $B_{100}=B / 100 \mu \mathrm{G}$ is the magnetic field in units of $100 \mu \mathrm{G}$.

The emission region has a spatial extent at $v \sim v_{\max }$ which is determined by diffusion and can be written as

$$
\Delta x \approx \sqrt{D\left(E_{\max }\right) \tau_{\text {loss }}\left(E_{\max }\right)} \approx 0.04 B_{100}^{-3 / 2} \mathrm{pc} .
$$

The typical thickness of the X-ray rims found through high resolution observations is of order $\sim 10^{-2} \mathrm{pc}$, thereby leading to predicting values of the magnetic field of $100-300 \mu \mathrm{G}$ downstream of the shock.

In Fig. 1 we show the X-ray brightness profile (histogram) of part of the rim of SN 1006. The thick lines refer to the

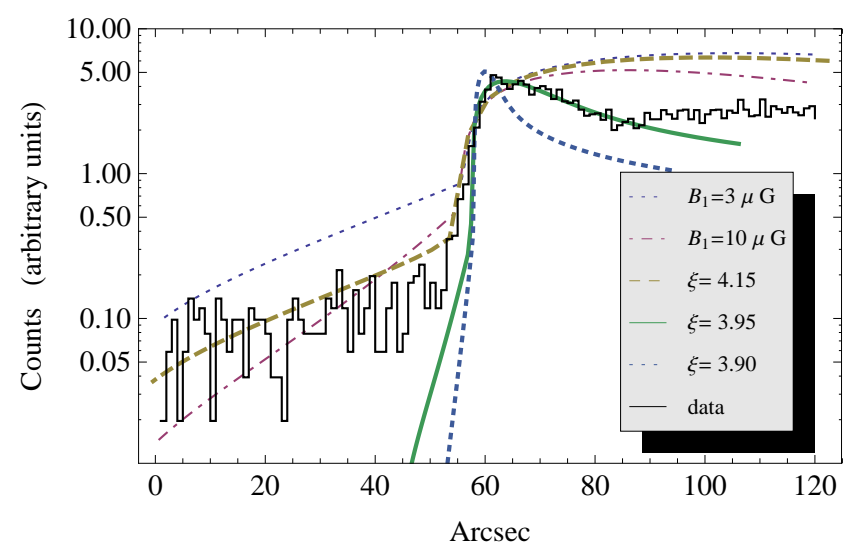

Figure 1: Radial profile of the emission in the $0.8-2.0 \mathrm{keV}$ band, extracted from a narrow strip around the rim of SN 1006 (thin black line). Overplotted are the theoretical predictions of NLDSA for 3 different injection efficiencies (thick lines) and those of test-particle theory for two different values of the preexisting turbulent magnetic field upstream (thin lines).

predictions of NLDSA[7] for three values of the injection efficiency (larger values of $\xi$ correspond to lower efficiencies). The thin lines are the predicted radial profiles in test-particle theory for two values of the upstream magnetic field (as indicated). Two pieces of information arise from this figure: 1) the narrow rims $(\sim 10-20$ arcsec) downstream can only be reproduced for efficient particle acceleration scenarios; 2) the predicted Xray emission from the precursor region drops below the background for the same cases in which the rims are present. In other words, the non-detection of the precursor X-ray emission might be additional evidence for efficient acceleration. This is due to the fact that the spatial extent of the precursor is reduced when magnetic fields are amplified by CR streaming.

Although very interesting, this interpretation is not totally unique: it could be that the magnetic field is not amplified upstream by CRs, but rather enhanced due to fluid instabilities downstream in quasi-perpendicular shocks [8]. In this case the non-detection of the upstream emission could be due to a dominantly perpendicular topology of the magnetic field lines.

\section{2. $\gamma$-ray observations}

A crucial step towards confirming or rejecting the SNR paradigm might be made through gamma-ray observations both in the TeV energy range, by using Cherenkov telescopes, and in the GeV energy range accessible to the Fermi and AGILE gamma-ray telescopes. Gamma radiation can be produced mainly as a result of inverse Compton scattering (ICS) of relativistic electrons on the photon background and in inelastic proton-proton scatterings with production and decay of neutral pions. In the last few years many SNRs have been detected both with Cherenkov and with gamma-ray telescopes but the present observational situation is rather puzzling. From one hand the idea that SNRs can accelerate hadrons has been confirmed, beyond any reasonable doubt, by the detection of a bump in the GeV spectrum of the remnant W44 by AGILE[9]. This bump is in fact interpreted as the threshold of the $\pi^{0}$ decay, pointing 
towards an hadronic origin of the $\mathrm{GeV}$ spectrum. On the other hand the maximum energy of protons inferred from the spectrum of W44 is $\sim 60-100 \mathrm{GeV}$, orders of magnitudes below the knee energy of the CR spectrum.

Moreover the gamma-ray spectrum of W44 as well as most spectra observed by Fermi (see [10, 11] for reviews) hint to rather steep spectra of accelerated particles $\left(\propto E^{-\gamma}\right.$, with $\gamma \sim$ $2.3-3$ ), which are not easy to accommodate in the context of NLDSA that predicts flat spectra, possibly even flatter than $E^{-2}$ at high enough energy[12].

The most likely explanation for this discrepancy might lie in a rather subtle detail of the DSA theory, namely that the velocity relevant for particle acceleration is the velocity of waves with respect to the plasma. Particles scatter with magnetic turbulence upstream and downstream af the shock, and feel an effective compression ratio which is given by

$$
r=\left(u_{1} \pm v_{A, 1}\right) /\left(u_{2} \pm v_{A, 2}\right),
$$

where $u_{1}\left(u_{2}\right)$ and $v_{A, 1}\left(v_{A, 2}\right)$ are the plasma and the wave speeds upstream (downstream), respectively, and the sign depends on the wave propagation direction. Usually the wave speed is negligible compared with the plasma velocity in the shock frame, but in the presence of magnetic field amplification this condition might be weakly violated. This is very bad news in that the spectral changes induced by this effect depend not only on the wave speed but on the wave polarization as well (which determines the signs in Eq. (5)). In [13, 14] the authors show that there are situations in which the spectral steepening can indeed be sufficient to explain the observed spectrum of CRs and required by Fermi data on some SNRs. In fact, assuming that the turbulence is due to Alfvèn waves induced by the streaming of CRs, than $v_{A, 1}=B_{1} / \sqrt{4 \pi \rho_{1}}$ is the upstream Alfvèn speed, to be taken with negative sign because waves propagate away from the shock, and $v_{A, 2} \approx 0$ because downstream we can expect efficient helicity mixing. If the magnetic field amplification is strong enough to produce $v_{A, 1} \simeq 0.2 u_{s h}$ than the particle spectral index becomes $\gamma=(r+2) /(r-1) \simeq 2.3$.

The main caveat in the previous argument is that steep spectra $(\gamma>2)$ imply low energy in the highest energy particles. But those particles are the only ones that, through the streaming instability, can produce turbulence with the right wavelength on which they scatter efficiently. If the energy density in those waves is not large enough the scattering is not efficient and particles can escape the system without reaching the required energy to explain the CR spectrum. The only way to test this mechanism is to develop consistent model of particle-wave interaction in the contest of NLDSA and compare the predictions with observations.

Indeed this idea has been successfully applied to the Tycho's remnant [15] which is, up to date, the only convincing SNR showing evidence for efficient CR acceleration from the gamma-ray observation. Tycho is a young type-I/a SNR detected in many wavebands, from radio up to TeV. In [15] the NLDSA has been coupled to magnetic field amplification (including the wave speed effect) and to the radiative processes in a self consistent way, in order to explain the observed multiwavelength spectrum. Fig. (2) shows the result. All data are

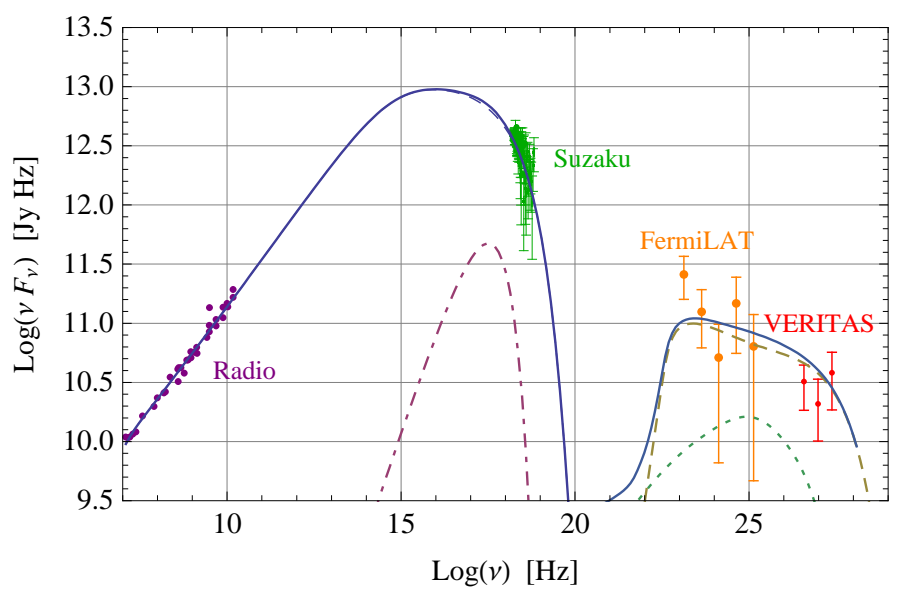

Figure 2: Spatially integrated spectral energy distribution of Tycho's SNR. The curves show synchrotron emission (thin dashed), thermal electron bremsstrahlung (dot-dashed), pion decay (thick dashed) and ICS (dotted) as calculated using NLDSA. The total sum is showed by the solid curve. The experimental data from Fermi-LAT and VERITAS include only statistical error at $1 \sigma$. The figure is from [15].

well explained by the model. The steepening induced by the wave speed with respect to the plasma results in a gamma-ray spectrum $\propto E^{-2.2}$ which well account for the GeV-TeV data. At the same time the streaming instability plus the shock compression results in a downstream magnetic field $\sim 300 \mu \mathrm{G}$ which well account for the integrated radio and X-ray emission as well as for their morphology. Remarkably the maximum energy inferred for accelerated protons is $\sim 500 \mathrm{TeV}$, only a factor 6 below the knee energy, while the total energy converted into accelerated particles is $\sim 12 \%$ of the bulk kinetic energy of the expanding shock, which is, again, what is required in order to explain the CR flux observed at Earth.

\subsection{Acceleration in partially neutral plasmas and Blamer emission}

Shocks produced by supernaova explosions are collisionless, which means that the fluid discontinuities are formed by electromagnetic instabilities while particle collisions are negligible because the low density of interstellar medium. On the other hand the interstellar medium itself is often only partially ionized. As we will explain in a while, the presence of neutrals can significantly modify the shock structure in a way that up to know has been completely neglected in the shock theory. As a consequence, the spectrum of accelerated particles can also be affected.

The neutral component interacts with the ionized component only through the processes of charge exchange and ionization. These two processes lead to exchange of energy and momentum between charged and neutral particles both upstream and downstream of the shock. In particular, neutrals that suffer a charge exchange downstream with shock-heated ions generate high velocity neutrals that have a finite probability of returning upstream. These neutrals might then deposit heat in the upstream plasma through ionization and charge exchange, thereby 
reducing the fluid Mach number. This phenomenon, leads to a reduction of the shock compression factor and to the formation of a neutral-induced precursor upstream of the shock [16]. The scale length of the precursor is determined by the ionization and charge exchange interaction lengths of fast neutrals moving towards upstream infinity. In the case of a shock propagating in the interstellar medium, the effects of ion-neutral interactions are especially important for shock velocities $<3000 \mathrm{~km} / \mathrm{s}$. What is remarkable is that, when we consider the acceleration of particles, the reduction of the sub-shock compression factor (induced by neutrals) to values $<4$ can result in a spectrum of accelerated particles steeper than $E^{-2}$. This effect is energy dependent and is more effective for particles whose diffusion length ahead of the shock is $\lesssim$ than the ionization or charge exchange interaction lengths.

The structure of shocks in presence of neutrals can be described using the kinetic theory where the coupling of neutral particles with ions and electrons through charge-exchange and ionization is described by the the Vlasov equation. In Fig. (3) we report a preliminary result of this effects: we computed the slope of accelerated particles in the test-paricle case considering a total density of 1 particle per $\mathrm{cm}^{3}$ with $50 \%$ of ionization fraction and an upstream magnetic field of $10 \mu \mathrm{G}$. We see that for shock speed $<3000 \mathrm{~km} / \mathrm{s}$ even particles at $1 \mathrm{TeV}$ can have a slope steeper than 2 .

In principle the presence of neutrals can provide an explanation for the steep spectra observed in $\mathrm{GeV}$ band from several SNRs (see \$3.2). Nevertheless these results should be taken with care because are computed in test particle theory; when the effect of CR will be included, non linear effects could change the conclusion even dramatically.

Apart of the effect that neutrals have on the shock dynamics, there is a second reason why they are worth to study. Hydrogen atoms that undergo charge exchange or collisional excitation produce Balmer and Lyman lines. These lines have been detected from several SNR shocks (especially Balmer) which, for this reason, are called Balmer-dominated shocks. What is remarkable is that Balmer emission can be used to infer some shock properties, including the acceleration efficiency, as we will explain in the following.

The emission lines typically have two components. The first one is a narrow line whose width is characteristic of the cold interstellar medium and results from direct excitation of neutral hydrogen atoms. The second component has a much broader line width, and arises from a second population of hydrogen atoms created by charge exchange process between cold preshock hydrogen and hot shocked protons. These hot atoms can be produced in an excited state or can be excited by subsequent collisions with protons or electrons. Hence the line width of the broad component is characteristic of the thermal velocity of the shocked protons.

The situation becomes more interesting in the presence of CRs: when the pressure of accelerated particles upstream of the shock generates the precursor, in the shock frame the bulk motion of ions is slowed down with respect to neutrals. Neutral atoms keep moving with the velocity that ions have at upstream infinity, $u_{s h}$, therefore a difference in bulk velocity between the two species arises. At the shock crossing, the ionized plasma suffers shock heating and compression while the neutral component does not feel the shock and keeps moving with speed $u_{s h}$ (with respect ot the shock frame). In the presence of CR acceleration, the temperature of the ions downstream is clearly lower that in the absence of CRs, simply because of energy conservation (there is now another component, CRs, into which the ram pressure $\rho_{0} u_{s h}^{2}$ can be channeled). Therefore one can expect that the width of the broad Balmer line is somewhat smaller than in the absence of CRs. This phenomenon has been recently observed in the SNR RCW86[17].

Measurements of the proper motion of the shock lead to a shock velocity of $u_{s h}=6000 \pm 2800 \mathrm{~km} / \mathrm{s}$, which in turn should imply a downstream temperature of $T_{2}=20-150 \mathrm{keV}$ or $T_{2}=12-90 \mathrm{keV}$ depending on whether protons and electrons reach or not thermal equilibrium downstream, and assuming that no $\mathrm{CR}$ acceleration is taking place. The temperature inferred from the width of the broad Balmer line is $T_{2}=19.2 \pm 1.1$ $\mathrm{keV}$. The authors interpreted this discrepancy as the result of effective CR acceleration at the SNR shock, with an estimated efficiency of $\gtrsim 15 \%$ considering an electron temperature $<70 \%$ of the proton temperature.

In CR modified shocks also the width of the narrow line should be modified. In fact some level of charge exchange is expected to take place in the precursor because of the difference in bulk velocity between the ionized plasma and the neutrals. The process is responsible for producing a population of warmer neutrals upstream. After shock crossing this leads to a narrow Balmer line which is broader than in the absence of a CR induced precursor. This effect was observed in several SNRs[18]. An independent signature of the same phenomenon was recently found in the Tycho's SNR[19]. The authors used the Hubble Space Telescope to measure the intensity of the broadened narrow Balmer line as a function of position around the shock and claim that there is substantial emission from the region in front of the shock. This finding could be either the signature of different bulk velocities between ions and neutrals (precursor) or of a different temperature of the two components. In this latter case, the ions could have been heated up due to the action of turbulent heating in the precursor.

\section{Conclusions}

We provided a short review of the particle acceleration theory around SNR shocks underlining the fact that accelerated particles cannot be passive spectators in the acceleration process, but they should have an important dynamical role. When the backreaction of those particle is taken into account, the theory makes several predictions which seems to be confirmed by recent observational findings. Probably the most streaking one is the detection of narrow X-ray filaments close to the shocks, which are interpreted as due to rapid electron synchrotron losses, supporting the idea that the magnetic field is strongly amplified as predicted by NLDSA.

On the other hand gamma-ray observations challenges the current theory because the observed spectra in the GeV-TeV 


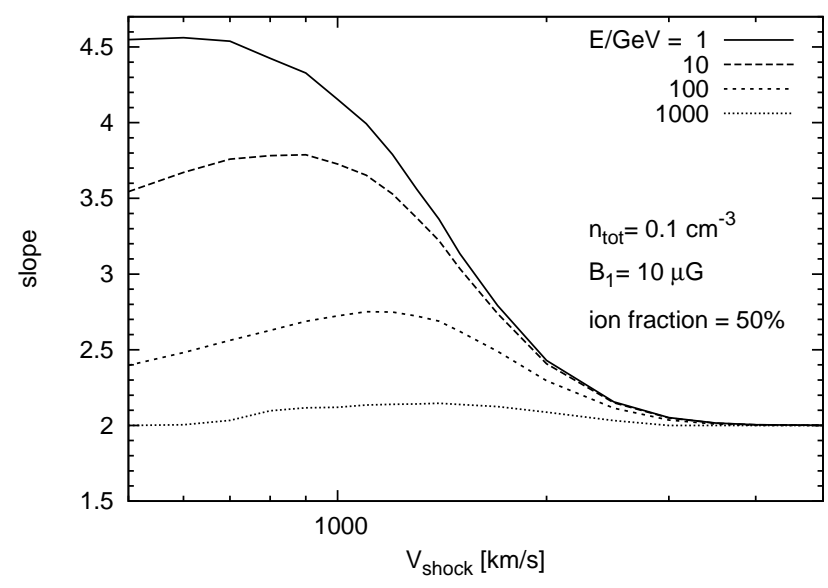

Figure 3: Slope of the spectrum of accelerated particles in case of shock propagating into a plasma with $50 \%$ ionization fraction and total density of 1 particle per $\mathrm{cm}^{-3}$. Calculations are performed in test particle limit for energies $E=1$, $100,100,1000 \mathrm{GeV}$, and as a function of the shock speed. The presence of neutrals produces a deviation from the standard slope equal to 2 .

range are typically steeper than what NLDSA predicts. Nevertheless we showed that this discrepancy can be accounted for at least in two different ways. One possibility is to take into account the finite velocity of waves responsible for particle scattering which, under certain circumstances, reduces the effective compression ratio felt by particles, resulting in steeper spectra. The second possibility involves the role of neutral particles which has never been properly taken into account in shock theory. We showed that neutrals can modify the shock structure through the processes of charge exchange and ionization with the ionized component, producing a neutral-induced precursor ahead of the shock. Also in this case the compression ratio can be remarkably reduced and steeper spectra are produced. Both these ideas still require to be tested in a full self-consistent theory.

The inclusion of neutrals in the shock theory has a second important consequence. Hydrogen atoms that undergo excitation and charge-exchange in the shock region produce Balmer and other optical lines. These lines can be used to infer the temperature of the shocked gas and also to reveal the presence of a precursor providing an estimate of the CR acceleration efficiency.

\section{References}

[1] A. R. Bell, MNRAS 182, 147 (1978)

[2] R. Blandford \& D. Eichler, Physics Reports 154, 1 (1987)

[3] P. O. Lagage and C. J. Cesarsky, Astron. and Astrophys. 125, 249 (1983)

[4] M. Malkov and L. Drury, Reports on Progress in Physics 64, 429 (2001)

[5] E. Amato and P. Blasi, MNRAS Letters 364, L76 (2005)

[6] D. Caprioli, P. Blasi, E. Amato \& M. Vietri, ApJL 679, L139 (2008)

[7] G. Morlino, E. Amato, P. Blasi and D. Caprioli, MNRAS Letters 405, L21 (2010)

[8] J. Giacalone and J. R. Jokipii, ApJL 663, L41 (2007).

[9] A. Giuliani et al. (AGILE collaboration), ApJL 742, L30

[10] S. Funk, Fermi-lat observations of young supernova remnants (2009)
[11] T. Tanaka, Fermi lat observations of supernova remnants interacting with molecular clouds (2009).

[12] D. Caprioli, P. Blasi and E. Amato, MNRAS 396, 2065 (2009)

[13] D. Caprioli, P. Blasi and E. Amato, arXiv astro-ph.HE 1011.6527 (2010)

[14] V. Ptuskin, V. Zirakashvili \& E.-S. Seo, ApJ 718, 31 (2010)

[15] G. Morlino \& D. Caprioli, MNRAS 538, 81, (2011)

[16] P. Blasi, G. Morlino, R. Bandiera, E. Amato \& D. Caprioli, ApJ 755, 121 (2012)

[17] E. A. Helder, D. Kosenko, \& J. Vink, ApJL 737, L46 (2011)

[18] J. Sollerman, P. Ghavamian, P. Lundqvist \& R. C. Smith, Astron. and Astrophys. 407, 249 (2003)

[19] J. J. Lee et al. ApJ 715, 146 (2010) 